\title{
Editorial
}

\section{Special issue on real-time and ubiquitous social semantics}

\author{
Alexandre Passant ${ }^{\mathrm{a}}$, Fabien Gandon ${ }^{\mathrm{b}}$ and Harith Alani ${ }^{\mathrm{b}}$ \\ ${ }^{a}$ DERI, NUI Galway, Ireland \\ E-mail: Alexandre.Passant@deri.org \\ ${ }^{\mathrm{b}}$ INRIA Sophia Antipolis, France \\ E-mail: Fabien.Gandon@inria.fr \\ ${ }^{\mathrm{c}} \mathrm{KMi}$, The Open University, UK \\ E-mail:h.alani@open.ac.uk
}

The Web has shifted from its initial document and librarian paradigm to an ecology of socially-generated data and services. Websites such as Twitter, Facebook, and FourSquare, emphasise the huge popularity of sharing information in real-time. In addition, the wealth and breadth of applications that exploit open social networking APIs to provide new services and functionalities are growing rapidly, enabling new ways to interact and browse this user-generated content.

At the same time, the deployment of networkenabled mobile devices, RFID and sensors, is realising the ubiquitous nature of social networks. More objects from our everyday life are getting connected to the Internet to become part of its applications, including the Web and social networking services. We are only starting to contemplate the potential of a wide Internet of things, but it is certain that in that new augmentation of our reality, the Semantic Web will be one of the cornerstones of interoperability.

Advances in the Semantic Web and Linked Data realms offer new capabilities for such paradigms, ranging from data integration to knowledge representation for such social data, objects, and service descriptions. However, many challenges remain to be addressed such as scalability, reasoning in dynamic con- texts, quality and provenance, privacy and security, multi-modal accesses, context capture and awareness, etc. Nevertheless, Semantic Web frameworks provide the means to support the architecture of such real-time social and ubiquitous platforms.

In this special issue, we present two papers that tackle the issues of real-time and ubiquitous Social Semantics. The first paper is by Matthew Rowe (Open University, UK) and Milan Stankovic (Hypios, France) where they used machine learning and semantics to automatically map Twitter feeds about a particular conference to the actual events taking place during that conference. The second paper is authored by Stefan Zander (University of Vienna, Austria) and Bernhard Schandl (Gnowsis.com, Austria) who developed a framework for mobile devices to efficiently retain some data from the Semantic Web to operate services offline. The framework they produced selectively replicates data from external sources using semantic descriptions of users' contextual information needs.

The field of real-time ubiquitous social semantics is still in its infancy, and we hope that this special issue will draw more attention to it and help focussing research and development on the various topics highlighted in this issue. 